\title{
Generalization of the algorithm of determining mass flow rate by measuring pressure in gas transportation systems
}

\author{
Edward Bondarev ${ }^{1, *}$, Igor Rozhin $^{1}$, and Kira Argunova ${ }^{1}$ \\ ${ }^{1}$ Institute of Oil and Gas Problems, Siberian Branch, Russian Academy of Sciences, 677980 Yakutsk, \\ Oktyabskaya, 1, Russia
}

\begin{abstract}
The current algorithm for calculating mass flow rate in gas production and transportation systems via outlet pressure measurements is generalized to the case when the inner cross section of pipe changes with time and is also to be determined while solving the general problem. The algorithm is recommended for identification of gas hydrate formation in the above-mentioned systems.
\end{abstract}

The formation and deposition of hydrates in pipelines is studied using quasi-stationary mathematical model [1-8]. In this model imperfect gas flow in pipes is described in the frame work of tube hydraulic and dynamics of hydrate formation - in the frame work of the generalized Stefan problem in which temperature of gas-hydrate phase transition depends on gas flow pressure. The model is closed by some phenomenological relations corresponding to the level of description of physical phenomena, so that the equations inevitably contain some constants determined on the basis of the selected model from additional information [9], which can be, for example, gas pressure and temperature measurements. In addition to the phenomenological constants, the model includes technological parameters, for example, mass flow rate of gas which is constant at stationary flow, that can also be determined from these measurements. This inverse problem of determining the coefficients of differential equations via some additional information about behavior of the solution is the subject of this paper, where upon the algorithms of determining the parameters of ordinary differential equations created in $[8,10,11]$ are generalized for the hydrate formation model proposed in [1-8]. An essential feature of this model is the determination of pipe cross-section that changes with time along with the calculation of gas temperature and pressure distribution via solving the Cauchy problem for equation which describes how dimensionless cross-section $S$ changes over time:

$$
\frac{d S}{d \tau}=b_{2} \frac{T_{\mathrm{e}}-T_{\mathrm{h}}(p)}{1-b_{2} \ln S}-b_{1} \sqrt{S}\left(T_{\mathrm{h}}(p)-T\right),
$$

In the intervals of pipeline where there is hydrate layer builds up, the heat transfer coefficient in equation (1) is calculated using the formula

\footnotetext{
*Corresponding author: bondarev@ipng.ysn.ru
} 


$$
\frac{\alpha_{1} d_{0}}{\lambda_{\mathrm{g}}}=0.023 \operatorname{Pr}^{0.43}\left(\frac{4 M}{\pi d_{0} \eta_{\mathrm{g}}}\right)^{0.8} \frac{1}{S^{0.9}}
$$

and ambient temperature $T_{\mathrm{e}}$ is replaced with equilibrium hydrate formation temperature $T_{\mathrm{h}}$.

Now we will set forth an algorithm for determining mass flow rate of gas from pressure measurements at the outlet, when cross-section varies along the length and in time due to the formation (dissociating) of hydrate layer. First note that the model proposed in [1-8] is sensitive to the input data due to its nature (nonisothermic process, real properties of the gas, cross-section of pipe that changes with time, heat exchange with environment). These items are discussed in detail in monographs $[8,11,12]$.

We define the zero approximation $M^{0}$ and calculate $p^{0}(x)$ and $T^{0}(x)$ following the method described in [11] and using the model proposed in $[2,8]$

$$
\frac{d p}{d x}=f_{1}(x, p, T, M), \quad \frac{d T}{d x}=f_{2}(x, p, T, M),
$$

where $f_{1}=-\frac{\sqrt{\pi} \psi M^{2}}{4 \rho_{\mathrm{g}} S^{2.5} S_{0}^{2.5}}, f_{2}=\frac{\pi d \alpha}{c_{p} M}\left(T_{\mathrm{e}}-T\right)+\varepsilon f_{1}, c_{p}-$ specific heat capacity of gas at constant pressure, $d$ - diameter of cross-section, $M$ - constant mass flow rate, $p$ - pressure, $S$ - dimensionless cross-section, $S_{0}$ - dimensional cross-section before formation of hydrates, $T$-temperature, $T_{\mathrm{e}}$ - temperature of surrounding frozen ground, $x$-coordinate along pipe axis, $\alpha$ - total heat transfer coefficient between gas and surrounding ground, $\rho_{g}-$ gas density, $\psi$-hydraulic resistance coefficient, $\varepsilon$ - throttling coefficient which can be determined from gas imperfection coefficient, depending on ratio of pressure and temperature to their critical values $p_{\mathrm{c}}$ and $T_{\mathrm{c}}$. Here it has the form of the Berthelot equation [13].

In the system (3) linearized with respect to the $(s+1)$-th approximation:

$$
\begin{gathered}
\frac{d p^{s+1}}{d x}=f_{1}^{s}+\left(\frac{\partial f_{1}}{\partial p}\right)^{s}\left(p^{s+1}-p^{s}\right)+\left(\frac{\partial f_{1}}{\partial T}\right)^{s}\left(T^{s+1}-T^{s}\right)+\left(\frac{\partial f_{1}}{\partial M}\right)^{s}\left(M^{s+1}-M^{s}\right), \\
\frac{d T^{s+1}}{d x}=f_{2}^{s}+\left(\frac{\partial f_{2}}{\partial p}\right)^{s}\left(p^{s+1}-p^{s}\right)+\left(\frac{\partial f_{2}}{\partial T}\right)^{s}\left(T^{s+1}-T^{s}\right)+\left(\frac{\partial f_{2}}{\partial M}\right)^{s}\left(M^{s+1}-M^{s}\right),
\end{gathered}
$$

substitute the solutions $p^{s+1}(x)$ and $T^{s+1}(x)$, expressed in terms of the sweep coefficients $C$ and $D$

$$
p^{s+1}=C_{1} M^{s+1}+D_{1}, \quad T^{s+1}=C_{2} M^{s+1}+D_{2},
$$

As a result, we obtain the following equations for these coefficients:

$$
\begin{aligned}
& \frac{d C_{1}}{d x}=\left(\frac{\partial f_{1}}{\partial p}\right)^{s} C_{1}+\left(\frac{\partial f_{1}}{\partial T}\right)^{s} C_{2}+\left(\frac{\partial f_{1}}{\partial M}\right)^{s}, \\
& \frac{d C_{2}}{d x}=\left(\frac{\partial f_{2}}{\partial p}\right)^{s} C_{1}+\left(\frac{\partial f_{2}}{\partial T}\right)^{s} C_{2}+\left(\frac{\partial f_{2}}{\partial M}\right)^{s},
\end{aligned}
$$




$$
\begin{aligned}
& \frac{d D_{1}}{d x}=\left(\frac{\partial f_{1}}{\partial p}\right)^{s}\left(D_{1}-p^{s}\right)+\left(\frac{\partial f_{1}}{\partial T}\right)^{s}\left(D_{2}-T^{s}\right)+f_{1}^{s}-\left(\frac{\partial f_{1}}{\partial M}\right)^{s} M^{s}, \\
& \frac{d D_{1}}{d x}=\left(\frac{\partial f_{1}}{\partial p}\right)^{s}\left(D_{1}-p^{s}\right)+\left(\frac{\partial f_{1}}{\partial T}\right)^{s}\left(D_{2}-T^{s}\right)+f_{1}^{s}-\left(\frac{\partial f_{1}}{\partial M}\right)^{s} M^{s},
\end{aligned}
$$

and the initial conditions

$$
C_{1}(0)=C_{2}(0)=0, D_{1}(0)=p_{0}, D_{2}(0)=T_{0} .
$$

After numerical solution of these Cauchy problems, using the condition at the outlet $p(L)=p_{\mathrm{y}}$ and relation (9), we find

$$
M^{s+1}=\frac{p_{\mathrm{y}}-D_{1}(L)}{C_{1}(L)},
$$

where $L$ is the length of the pipeline, $p_{\mathrm{y}}$ is the outlet pressure.

Now the algorithm for the numerical solution of the conjugate problem of heat transfer between gas pipeline and frozen ground can be described as follows [2]:

1. Find the zero approximation of pressure distribution, which correspond to the simplified model of isothermal flow of ideal gas without the formation of a hydrate $(S=1)$ :

$$
p^{0}(x)=\left(p_{0}^{2}-\frac{\sqrt{\pi} \psi R x T_{0}\left(M^{0}\right)^{2}}{2 S_{0}^{2.5}}\right)^{1 / 2}
$$

2. Set initial values of sweeping coefficients according to equations (11) and then find the solutions (6).

3. By solving the Cauchy problem for system (7)-(10) find $C_{1}(x)$ and $D_{1}(x)$.

4. According to equation (12) determine the mass flow rate $M^{s+1}$.

5. By substituting $M^{s+1}$ into relations (6) find $p^{s+1}(x)$ and $T^{s+1}(x)$.

6. Steps 2 - 5 are repeated until the required accuracy is achieved. This parameter depends on characteristics of the object where measurements are performed. The most important here is the accuracy of measuring apparatus.

7. From equation (1) taking a step in time find a new value of cross-section. Coordinate $x$ enters this equation as a parameter.

8. Determine the temperature distribution in surrounding ground solving the initial boundary value problem for equation of heat conduction using the enthalpy method [6].

At each time step points 3 - 9 are repeated.

The calculations were carried out with the following initial data: $c_{p}=2300 \mathrm{~J} /(\mathrm{kg} \cdot \mathrm{K})$, $d_{0}=1.4 \mathrm{~m}, L=100 \mathrm{~km}, \quad q_{\mathrm{h}}=510 \mathrm{~kJ} / \mathrm{kg}$, operating pressure $p_{0}=9.8 \mathrm{MPa}$, inlet temperature $T_{0}=282.15 \mathrm{~K}$, heat exchange coefficient with thermal insulation $\alpha_{0}=0.145 \mathrm{~W} /\left(\mathrm{m}^{2} \cdot \mathrm{K}\right), \quad \psi=0.02, \quad \lambda_{\mathrm{g}}=0.0307 \mathrm{~W} /(\mathrm{m} \cdot \mathrm{K}), \quad \lambda_{\mathrm{h}}=1.88 \mathrm{~W} /(\mathrm{m} \cdot \mathrm{K})$, $\eta_{\mathrm{g}}=1.3 \cdot 10^{-5} \mathrm{~Pa} \cdot \mathrm{s}, \rho_{\mathrm{h}}=920 \mathrm{~kg} / \mathrm{m}^{3}$, initial ground temperature $T_{\mathrm{fr}}=271.15 \mathrm{~K}$, temperature of ground thawing-freezing $T_{\mathrm{ph}}=273.15 \mathrm{~K}$, latent heat of ice-water phase transition $q_{\mathrm{ph}}=334.4 \mathrm{~kJ} / \mathrm{kg}$; for lightly icy sandy clays thermal conductivity and volume heat 
capacities in thawed and frozen states: $\lambda_{\text {liq }}=1.6 \quad \mathrm{~W} /(\mathrm{m} \cdot \mathrm{K}), \quad \lambda_{\mathrm{s}}=1.7 \quad \mathrm{~W} /(\mathrm{m} \cdot \mathrm{K})$, $C_{\text {liq }}=2.8 \cdot 10^{6} \mathrm{~J} /\left(\mathrm{m}^{3} \cdot \mathrm{K}\right), C_{\mathrm{s}}=2.1 \cdot 10^{6} \mathrm{~J} /\left(\mathrm{m}^{3} \cdot \mathrm{K}\right)$, respectively; density $\rho=1760 \mathrm{~kg} / \mathrm{m}^{3}$, moisture content $\omega=0.233$. The gas constant $R=453.524 \mathrm{~J} /(\mathrm{kg} \cdot \mathrm{K})$, critical parameters $p_{\mathrm{c}}=4.5 \mathrm{MPa}$ and $T_{\mathrm{c}}=195.075 \mathrm{~K}$, coefficients $a=10.73 \mathrm{~K}$ and $b=117.979 \mathrm{~K}$ were found for natural gas from the Chayandinskoye field. The pipes made of K60 steel with wall thickness $0.032 \mathrm{~m}$ and heat conductivity $68 \mathrm{~W} /(\mathrm{m} \cdot \mathrm{K})$ have burial depth $1.5 \mathrm{~m}$ above the top of pipe. Pressure at the inlet of pipeline ranged from 6.6 MPa to $7 \mathrm{MPa}$.

In all instances the proposed algorithm required $3-6$ iterations for the calculations of mass flow rate with a relative accuracy of $0.1 \%$ were.

Let's analyze the calculation results presented in Fig. 1 - Fig. 6. We first recollect that for conjugated formulation the change in ground temperature due to its heat exchange with gas in pipe is taken into account, for non-conjugated one it is assumed to be equal to initial one $(268.15 \mathrm{~K})$.

First of all we note that dynamics of hydrate formation and their distribution along the pipeline are practically unaffected by changes in temperature field of the surrounding ground (see surfaces 1 and 2 in Fig. 1). This is explained by rather rapid hydrates formation (less than 4 days) which cause complete blockage of the outlet cross-section.

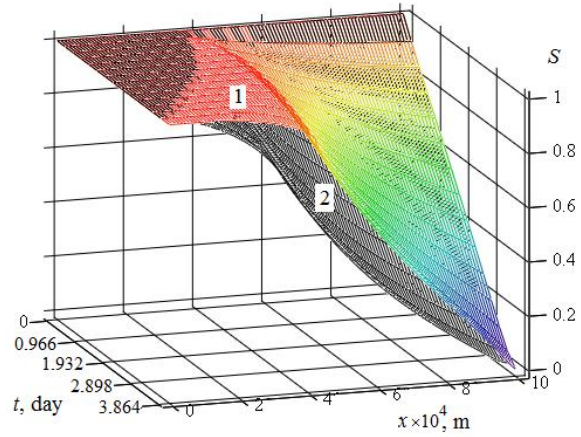

Fig. 1. The change of cross-section along pipeline and in time at $p_{\mathrm{y}}=6.6 \mathrm{MPa}$ :

1 - conjugated formulation, 2 - nonconjugated formulation

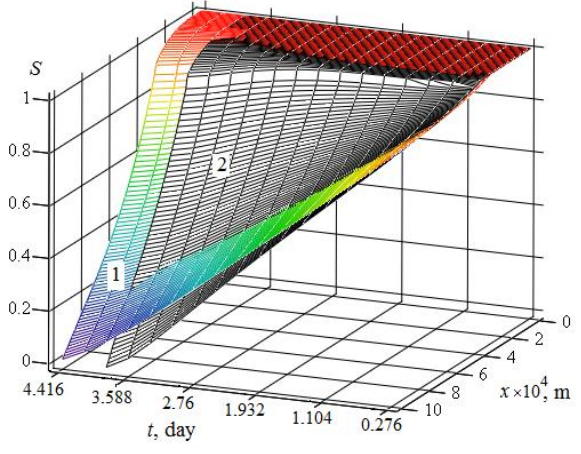

Fig. 2. The change of cross-section along pipeline and in time for conjugated formulation: $1-p_{\mathrm{y}}=7 \mathrm{MPa}, 2-p_{\mathrm{y}}=6.6$

$\mathrm{MPa}$

Fig. 2 illustrates the effect of the pressure drop along pipeline at the time of complete blockage. It is important to note here that for conjugated formulation the growth of hydrate layer, especially in the initial part of gas pipeline, occurs less intensively than for nonconjugated one. At the same time, the dynamics of this layer growth depends more on outlet pressure than on ground temperature (compare curves 1 - 4 in Fig. 2).

Let's compare the analyzed variants with the flow of completely dried gas, when the formation of hydrates in pipeline does not occur. First, we estimate the effect of hydrate formation on gas temperature. In Fig. 3 is clearly visible that gas temperature during the hydrate formation is lower than temperature of dried gas along the entire length of pipeline. At the initial part of pipeline $(30 \mathrm{~km})$ the difference is approximately $25 \mathrm{~K}$ (compare curves 6 and 3).

Such a change is explained by the dynamics of pressure distribution along pipeline and by the corresponding decrease of gas temperature due to throttling (Fig. 4). In this figure, surface 1 corresponds to pressure distribution during transportation of completely dry gas, which fully agrees with physics of the process, that is, practically stationary pressure distribution with slow temperature change due to heat exchange with the ground. However, 
when hydrate is formed, pressure in different parts of pipeline changes in accordance with the change of cross-section: it decreases slightly in the initial part (approximately $30 \mathrm{~km}$ ), and then very significantly (see surfaces 1 and 2 in Fig. 4). This tendency is fully reflected in the character of surface 2 in Fig. 4.

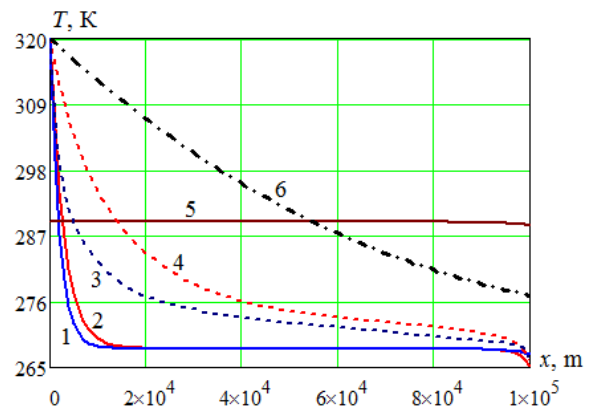

Fig. 3. Temperature variation along pipeline at the end ofcalculations: $1,3,6-p_{\mathrm{y}}=7 \mathrm{MPa} ; 2,4-$ $p_{\mathrm{y}}=6.6 \mathrm{MPa} ; 1,2$ - non-conjugated formulation; 3, 4, 6 - conjugated formulation; 5 - equilibrium temperature of hydrate formation; 6 - without formation of hydrates.

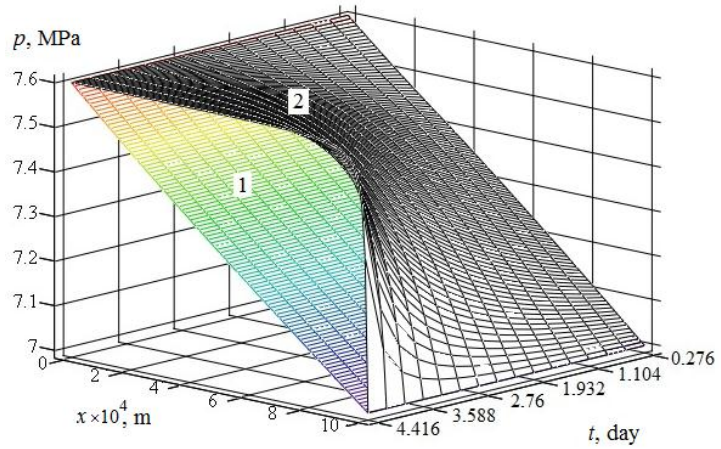

Fig. 4. The change of gas pressure along pipeline and in time at $p_{\mathrm{y}}=7 \mathrm{MPa}$ (conjugated formulation): 1 - without formation of hydrates, 2 - with formation of hydrates

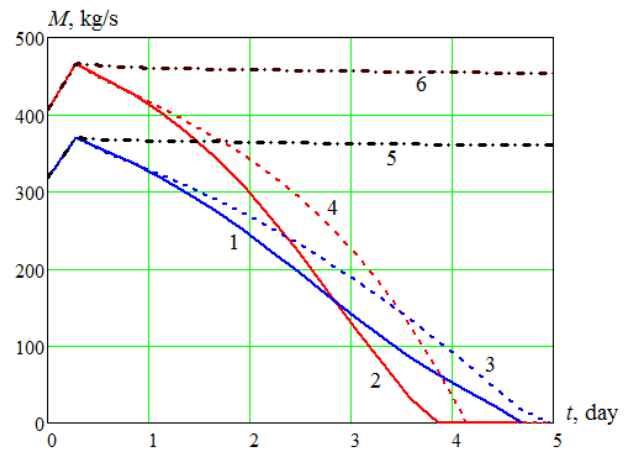

Fig. 5. Dynamics of mass flow rate: 1,3 and $5-p_{\mathrm{y}}=7 \mathrm{MPa} ; 2,4$ and $6-p_{\mathrm{y}}=6.6 \mathrm{MPa}$;

1 and 2 - non-conjugated formulation; 3 and 4 - conjugated formulation; 5 and 6 - conjugated formulation without formation of hydrates

Fig. 5 illustrates the effect of pressure drop and hydrate formation on the dynamics of mass flow rate of gas, that is, on the capacity of pipeline. First of all, here we note that for 
all computational options mass flow rate in a short initial period increases slightly due to the cooling of gas during its heat exchange with the ground. Then, for dry gas it remains almost constant, but in the case of hydrate layer formation it decreases to zero in a relatively short time. However, the rate of decrease is almost independent of heat exchange with the ground (compare the pairs of curves 1, 3 and 2, 4 in Fig. 5). At the same time, it depends ambiguously on pressure drop (compare pairs of curves 1, 2 and 3, 4 in Fig. 5), although this effect can be explained by the fact that increase of pressure drop corresponds to increase of the initial mass flow rate.

The proposed generalized algorithm for solving the inverse problem of determining mass flow rate of gas with time-varying pipeline cross-section by pressure measurements revealed that hydrate formation leads to changes in dynamics of pressure and temperature distribution along pipeline. It is shown that the time of formation of hydrate plug is determined, to the greatest extent, by the pressure drop across pipeline and, to a lesser extent, by ground temperature. For the parameters corresponding to a section of the modern main gas pipeline of $100 \mathrm{~km}$ length, it is 4 to 5 days.

\section{References}

1. E. A. Bondarev, V. I. Vasil'ev, A. F. Voevodin, N. N. Pavlov, A. P. Shadrina, Thermohydrodynamics of gas production and transportation systems, 272 (Novosibirsk: Nauka. Sibirskoe otdelenie, 1988) [in Russian]

2. E. A. Bondarev, I. I. Rozhin, K. K. Argunova, Journal of Applied Mechanics and Technical Physics, 58 (5), 853 (2017)

3. E. A. Bondarev, I. I. Rozhin, K. K. Argunova, Journal of Mining Institute, 228, 705 (2017)

4. K. K. Argunova, E. A. Bondarev, I. I. Rozhin, Earth's Cryosphere, 15 (2), 65 (2011)

5. E. A. Bondarev, K. K. Argunova, Information and Mathematical Technologies in Science and Management: Proceedings of 14th Baikal All-Russian Conference. Part 3, 41 (ISEM SO RAN, Irkutsk, 2009) [in Russian]

6. E. A. Bondarev, I. I. Rozhin, K. K. Argunova, Journal of Engineering Physics and Thermophysics, 87(4), 900 (2014)

7. E. A. Bondarev, I. I. Rozhin, A. F. Voevodin, K. K. Argunova, Problems of complex systems' optimization: Proceedings of 12th International Asian School-seminar, 79 (2016) [in Russian]

8. E. A. Bondarev, A. F. Voevodin, Solution of pipe hydraulics problems in natural gas production and transportation systems (Izdatel'stvo SO RAN, Novosibirsk, 2017) [in Russian]

9. I. I. Blekhman, A. D. Myshkis, Ya. G. Panovko, Applied mathematics: subject, logic and approaches, 270 (Naukova dumka, Kiev, 1976) [in Russian]

10. E. A. Bondarev, A. F. Voevodin, M. A. Kanibolotskii, E. A. Metlyaeva, Izv. Akad. Nauk SSSR. Energetika i transport., 1, 143 (1977) [in Russian]

11. E. A. Bondarev, A. F. Voevodin, V. S. Nikiforovskaya, Methods of identification of mathematical models of hydraulics (Izdatel'skii dom SVFU, Yakutsk, 2014) [in Russian]

12. O. F. Vasil'ev, E. A. Bondarev, A. F. Voevodin, M. A. Kanibolotskii, Nonisothermal gas flow in pipes (Nauka, (Novosibirsk, 1978) [in Russian]

13. I. I. Vukalovich, I. I. Novikov, Equations of real gas state, 343 (Gosenergoizdat, Moscow-Leningrad, 1948) [in Russian] 